\title{
Criminologie
}

\section{Trafics de drogues et criminalité organisée : une relation complexe}

\section{Michel Kokoreff}

Volume 37, numéro 2, automne 2004

Délinquance et réussite

URI : https://id.erudit.org/iderudit/010703ar

DOI : https://doi.org/10.7202/010703ar

Aller au sommaire du numéro

\section{Éditeur(s)}

Les Presses de l'Université de Montréal

ISSN

0316-0041 (imprimé)

1492-1367 (numérique)

Découvrir la revue

Citer cet article

Kokoreff, M. (2004). Trafics de drogues et criminalité organisée : une relation complexe. Criminologie, 37(2), 9-32. https://doi.org/10.7202/010703ar
Résumé de l'article

Les trafics de drogues constituent un objet sociologique mal identifié, alors même que leur développement alimente les débats publics sur l'insécurité, en France comme dans la plupart des pays occidentaux. Cette ambiguïté, si elle résulte de facteurs tant idéologiques et institutionnels que méthodologiques, traduit aussi la complexité des formes sociales de trafics trop souvent réduites à leur plus simple expression. Deux sources d'hétérogénéité peuvent être décrites : l'hétérogénéité des trafics d'un côté et l'hétérogénéité de leur traitement par la machine judiciaire de l'autre. Cet article s'attache plus particulièrement aux trafics d'envergure, c'est-à-dire à ces formes hybrides et peu explorées entre entreprises multinationales et commerces de rue. Il s'attache à montrer l'écart entre, d'une part, les pratiques considérées dans toute leur complexité individuelle, organisationnelle et sociale, et, d'autre part, leur construction par les pratiques des policiers et des magistrats, et leur interprétation plus globale dans le langage du droit, elles-mêmes déterminées par l'évolution des nouvelles procédures et des politiques.
Ce document est protégé par la loi sur le droit d'auteur. L'utilisation des services d’Érudit (y compris la reproduction) est assujettie à sa politique d'utilisation que vous pouvez consulter en ligne.

https://apropos.erudit.org/fr/usagers/politique-dutilisation/ 


\title{
Trafics de drogues et criminalité organisée: une relation complexe ${ }^{1}$
}

\author{
Michel Kokoreff \\ Maître de conférences à l'Université Paris V \\ Chercheur au Cesames, CNRS-INSERM \\ France \\ michel.kokoreff@paris5.sorbonne.fr
}

RÉSUMÉ - Les trafics de drogues constituent un objet sociologique mal identifié, alors même que leur développement alimente les débats publics sur l'insécurité, en France comme dans la plupart des pays occidentaux. Cette ambiguïté, si elle résulte de facteurs tant idéologiques et institutionnels que méthodologiques, traduit aussi la complexité des formes sociales de trafics trop souvent réduites à leur plus simple expression. Deux sources d'hétérogénéité peuvent être décrites: l'hétérogénéité des trafics d'un côté et l'hétérogénéité de leur traitement par la machine judiciaire de l'autre. Cet article s'attache plus particulièrement aux trafics d'envergure, c'est-à-dire à ces formes hybrides et peu explorées entre entreprises multinationales et commerces de rue. Il s'attache à montrer l'écart entre, d'une part, les pratiques considérées dans toute leur complexité individuelle, organisationnelle et sociale, et, d'autre part, leur construction par les pratiques des policiers et des magistrats, et leur interprétation plus globale dans le langage du droit, elles-mêmes déterminées par l'évolution des nouvelles procédures et des politiques.

ABSTRACT • Drug trafficking is a poorly identified sociological object, even though its development feeds debates about crime, in France as in the majority of Western countries. This ambiguity, resulting from ideological and institutional as well as methodological factors, also translates the complexity of the social forms of trafficking, which are too often reduced to their most simple expression. Two sources of heterogeneity can be described: the heterogeneity of the trafficking on one side, and the heterogeneity of its treatment by the legal system on the other. This article discusses large scale trafficking, that is to say, the hybrid forms little explored between multinational drug trafficking and trade on the street. The author attempts to show the

1. L'auteur souhaite remercier les lecteurs de la revue pour les remarques et suggestions qu'ils ont bien voulu apporter à une première version de ce texte. 
difference between the practices considered in their individual, organisational and social complexity, and their construction by the practices of the police officers and magistrates, including their more total interpretation in legal language, themselves determined by the evolution of the new procedures and policies.

Que savons-nous des trafics de stupéfiants d'envergure et de ceux qui s'y trouvent engagés pour en faire - au moins un temps - leur métier? $\mathrm{Si}$, des deux côtés de l'Atlantique en particulier, les enquêtes ne manquent pas sur les commerces du «coin de la rue» et autres «deals de cité $»^{2}$, pour en proposer de minutieuses descriptions à partir d'approches micro-locales, on sait, par contre, peu de choses de la sociologie des formes les plus professionnalisées de trafics ${ }^{3}$, qu'il s'agisse de leur histoire et de leur morphologie sociale ${ }^{4}$, de la composition des réseaux, ou encore des trajectoires biographiques de leurs principaux acteurs ${ }^{5}$. La distinction entre «trafic local» et «trafic international», qui s'impose le plus souvent avec l'éclat de l'évidence en France, est elle-même un indice de ce déficit de savoirs. Prédéfinies selon des critères qui relèvent plus du travail policier que de l'analyse sociologique, ces catégories sont, en effet, loin d'être homogènes; elles recouvrent une diversité de formes sociales souvent réduites à leur plus simple expression que viennent symboliser des figures emblématiques (de Scarface à Pablo Escobar, et des dealers des banlieues aux immigrés clandestins). En outre, ces catégories renvoient à une approche statique qui contribue à occulter les dynamiques de transformation sous-jacentes à ces activités délictueuses.

Divers facteurs peuvent expliquer un tel constat. On peut y voir une non-volonté de savoir de l'appareil d'État en ce qui concerne la question des drogues en général et celle des marchés illicites en particulier,

2. Citons, notamment, Adler (1985), Williams (1990), Bourgois (1992 et 2001), Fernandez (2002), Jamoulle (2002).

3. Divers travaux ont souligné la similitude des logiques de l'économie légale et de l'économie illégale du point de vue de ses formes d'organisation dans l'échange. L'idée n'est pas nouvelle puisqu'il y a près de trente ans, Preble et Casey (1969) avaient déjà montré tout l'intérêt de considérer l'usage de drogue comme style de vie, et Letkermann (1973), le crime comme travail, c'est-à-dire en termes de spécialisation, de professionnalisme, d'apprentissage et de satisfaction personnelle. Sur le trafic comme travail, voir plus récemment Bachmann et Coppel (1989), Joubert et Bouhnik (1992), Ruggiero et South (1996).

4. Voir notamment, Reuter et al. (1991), Ruggiero (1998), Schiray et al. (2000).

5. On pourra se reporter à l'autobiographie de Marks (2000) et aux analyses qu'en propose Morselli (2001). 
champ peu exploré par les sciences sociales en France jusqu'au tournant des années 1990 (Ehrenberg, 1992; Faugeron et Kokoreff, 1999). On peut aussi y lire la pente des politiques publiques qui, pour des raisons tant juridiques (la complémentarité incertaine des procédures, et les obstacles rencontrés, en vue d'une coopération des services) que politiques (le transfert de compétences et la promotion de nouveaux pôles de gouvernance urbaine), est de privilégier l'échelon local au nom d'une gestion de proximité de la sécurité publique plutôt que de s'attaquer en amont aux organisations criminelles - laissant croire à la possibilité de faire d'une pierre deux coups (Robert, 2002) ${ }^{6}$. S'ajoutent des facteurs d'ordre méthodologique: ce sont tout à la fois la nature des activités incriminées, les profits colossaux qu'elles sont susceptibles de générer, la violence systémique qui les entoure, la collusion des intérêts, ou encore les difficultés d'accès aux «terrains» et aux «bons informateurs», qui constituent autant d'obstacles non négligeables à l'objectivation. En un sens, les pouvoirs publics et les acteurs institutionnels d'un côté, les professionnels de l'économie illégale de l'autre, pour des raisons opposées, font eux-mêmes du secret et des barrières une condition de leur activité (Boltanski et Chiapello, 1999: 441 et 746).

Il en résulte un trou structurel entre les représentations collectives et la connaissance des faits sociaux, au moment même où la lutte contre

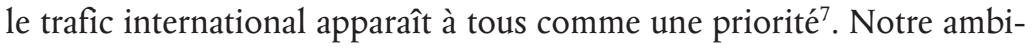
tion est moins de combler ces lacunes que d'interroger les liens entre les diverses formes sociales de trafic. Selon quelles logiques et quelles modalités ces marchandises illicites «font réseaux », transitent et s'échangent, produisent de la valeur? Des réseaux d'importation organisés

6. Cela explique en partie le retour sur le devant de la scène du thème des «mafias». À en croire nombre d'acteurs (politiques, institutionnels, sociaux) et d'experts en matière de sécurité intervenant dans le débat public, le développement d'une économie dite «souterraine» dans les banlieues et les quartiers populaires engendrerait des formes d'organisation et un climat de «violence» de type mafieux. Cette rhétorique renvoie à des enjeux idéologiques évidents: elle participe d'une stratégie de relégitimation de l'État passant par une «reconquête» de ces territoires qu'il a lui-même contribué à délaisser... Sur le terrain, les situations observables sont différentes: les pratiques des services de police et de gendarmerie consistent plus à poursuivre les «dealers» (petits revendeurs) que les «grosses têtes» (grossistes ou semi-grossistes); et cela, à travers des actions qui ciblent davantage des points de territoire que des réseaux.

7. Comme le constate Castells (1999: 195) dans l'analyse qu'il consacre à la «filière perverse», c'est-à-dire aux activités criminelles en réseaux internationaux: tout le monde souligne l'importance du phénomène, mais les chercheurs hésitent à l'étudier «au motif que les données manquent de fiabilité et que la recherche du sensationnel biaise trop souvent l'analyse». 
jusqu'aux territoires de revente dans les grandes métropoles, y a-t-il discontinuité des niveaux ou continum de positions? En d'autres mots, comment s'opère l'emboîtement entre des échelles territoriales ${ }^{8}$, des modes d'organisation et des trajectoires hétérogènes?

Afin d'explorer ces questions, on s'intéressera à des réseaux de trafic internationaux, et plus particulièrement aux filières d'importation du cannabis depuis le Maroc via l'Espagne jusqu'en France. Formes hybrides entre entreprises multinationales et trafics de rue, ces filières-réseaux ne se confondent ni avec les organisations criminelles classiques (les différentes «mafias» et autres «cartels»), que l'on persiste à présenter à partir d'un modèle centralisé et pyramidal ${ }^{9}$, ni avec les «circuits courts » (revente à la sauvette, trafics de fourmis, etc.), dont on a tendance à majorer tantôt la précarité (par misérabilisme), tantôt le degré d'organisation et de violence (par effet de méconnaissance). Par contre, on posera que ces réseaux caractérisent des formes originales de circulation des richesses dont l'intelligibilité va bien au-delà des drogues illicites.

$\mathrm{Si}$, par conséquent, ces formes possèdent leur logique propre, elles sont aussi produites par l'évolution des procédures judiciaires et des politiques publiques. En France, de nouvelles dispositions contre le trafic de stupéfiants ont été adoptées le 16 décembre 1992 et ajoutées au code pénal. Ces dispositions ont conduit à qualifier de crime les formes les plus professionnalisées de trafics, telles que «la direction ou l'organisation d'un groupement en vue de la commission d'infraction à la législation sur les stupéfiants, la production ou la fabrication illicite de ces derniers, l'importation et l'exportation commises en bande organisée». Afin d'éviter de provoquer une criminalisation excessive, le législateur s'est toutefois attaché à conserver des qualifications correctionnelles, et il a souhaité que soient créées des cours d'assises spéciales en raison de la complexité de ces affaires. En réalité, cette procédure criminelle a été peu appliquée. Et lorsqu'elle l'a été, les condamnations n'ont pas été à la hauteur des espérances. Le paradoxe est bien là : le but était de réprimer mieux, le résultat est de ne pratiquement pas réprimer à ce niveau - ou très inégalement selon les juridictions.

8. On s'inspire des analyses stimulantes de Tarrius (1997) et de Péraldi (2002).

9. L'Observatoire géopolitique des drogues (OGD) - organisme indépendant aujourd'hui disparu - a bien montré que, loin des discours officiels et des représentations collectives présupposant l'homogénéité du «crime organisé», l'observation des pratiques criminelles reflétait, au contraire, un monde éclaté (OGD, 1995). 
Ainsi, cet article décrit deux sources d'hétérogénéité: hétérogénéité des trafics d'un côté, hétérogénéité de leur traitement de l'autre. Il s'attache à montrer le décalage entre, d'une part, les pratiques considérées dans toute leur complexité individuelle, organisationnelle et sociale, et, d'autre part, leur construction par les pratiques des policiers et des magistrats et leur interprétation plus globale dans le langage du droit, elles-mêmes déterminées par l'évolution des nouvelles procédures et des politiques. L'analyse a pour arrière-plan une longue enquête de terrain menée dans certains quartiers d'habitat social constituant une "plaque tournante» du trafic de cannabis et d'héroïne dans la proche banlieue parisienne ${ }^{10}$. Déjà à l'échelle de ces espaces intermédiaires que sont les réseaux de quartiers ou de cités, les zones situées entre plusieurs communes ou départements, on observe des formes de trafics professionnalisées, au sens où elles s'appuient sur une division du travail et des rôles, une transmission des compétences et des ressources, un climat de tension dans ces territoires, qui en assurent la pérennité. Le néo-prolétariat des trafics dans les caves ou les cages d'escalier est dirigé, pour ne pas dire exploité, par des semi-grossistes dont les méthodes de travail et les chiffres d'affaires relèvent bien des logiques des économies informelles criminelles (Ruggioro, 1998). Bref, comme l'arbre ne saurait cacher la forêt, les «petits» ne doivent pas cacher les «gros». Mais c'est, précisément, aux niveaux supérieurs et à d'autres échelles qu'il s'agit de porter notre regard en s'appuyant, ici, sur une enquête collective effectuée au sein de trois territoires et juridictions situés dans la métropole lilloise (Nord), dans les Hauts-de-Seine et en Seine-Saint-Denis ${ }^{11}$.

\section{Les formes sociales des trafics en "bande organisée»}

Ces réseaux de trafics assurent l'acheminement par centaines de kilos de produits stupéfiants sur le territoire national. Ils permettent de comprendre le fonctionnement de filières d'importation de cannabis jusqu'en

10. Voir Duprez et Kokoreff (2000), Kokoreff (2003).

11. L'enquête a porté sur un corpus d'une quinzaine d'affaires d'importation de cannabis auxquelles s'est ajouté un corpus d'une cinquantaine d'affaires portant sur des formes plus localisées de trafic. Ces données ont été complétées, d'une part, par des entretiens avec divers acteurs (magistrats, policiers, avocats) et des observations d'audience, d'autre part, par des entretiens biographiques avec des entrepreneurs de la drogue. (Voir Duprez et al., 2001). On présentera ici essentiellement le volet concernant le tribunal de Nanterre. Les conditions dans lesquelles la cueillette des données judiciaires a été possible sont présentées en annexe. 
France et dans d'autres pays de la communauté européenne (Belgique, Hollande, Italie). Ainsi, ces marchandises illicites trouvent un débouché sur divers «marchés urbains illicites» (Lacoste et Tremblay, 1999), qu'il s'agisse d'agglomérations comme Paris, Bordeaux, Toulouse, ou de villes comme Rouen, Lille ou Dijon. Les dossiers judiciaires constituent de la sorte un apport assez riche en ce qu'ils livrent tout un ensemble de données permettant de mieux cerner la circulation des marchandises (logistique, distribution par les semi-grossistes, rapatriement de l'argent, etc.), les fonctions "périphériques» (maquillage des voitures, hébergement, comptage de l'argent, réseau de blanchiment, etc.) et les savoirfaire (savoir recruter, savoir circuler, savoir opérer des placements, etc.).

C'est aussi qualitativement que ces affaires témoignent des flux de trafic. L'Espagne est, depuis les années 1980, une tête de pont pour toute l'Europe en matière de drogues: le cannabis en provenance du Maroc et la cocaïne d'Amérique latine et des Antilles y transitent. On y trouve des réseaux anglais, allemands, italiens, français. Le sud de l'Espagne est devenu la base de repli d'un certain nombre de délinquants et de trafiquants notoires, souvent en «cavale». Il est vraisemblable que les commanditaires de ces trafics se connaissent de par leur passé en matière de délinquance et de leur localisation résidentielle. Peut-on déduire de ces liens d'interconnaissance une stratégie commerciale concertée? Cela revient à faire l'hypothèse sinon d'une organisation centralisée, du moins d'une «régulation» des marchés à cette échelle, et d'un moment donné, c'est-à-dire au milieu des années 1990.

\section{Profils et trajectoires}

Pour mieux apprécier l'assise sociale de ces réseaux, on utilisera le corpus d'affaires criminalisées et correctionnalisées constitué par les soins de Monique Weinberger à partir d'affaires jugées au tribunal de grande instance de Bobigny ${ }^{12}$. C'est tout d'abord la structure de la population traitée qui ressort. Dans ce corpus, il s'agit d'une population plus âgée (36 ans en moyenne contre 28 ans pour le trafic local, mais avec une palette d'âge assez étendue allant de la vingtaine à la soixantaine). Mieux

12. Il s'agit en fait d'un double corpus; le premier, daté de 1999, réunit 33 affaires impliquant 284 prévenus, dont 15 renvoyaient à des incriminations en bande organisée et représentaient 137 prévenus, et 18 au marché local pour 147 prévenus; le second, datant de 1994, est constitué, à titre d'élément de comparaison, de 30 affaires de trafic local qui impliquaient 157 personnes (voir Duprez et al., 2001 : 128-130). 
insérée socialement (68\% des personnes vivent en couple contre $41 \%$, et $45 \%$ sont salariées contre $37 \%$ ), cette population possède un niveau d'études plutôt qualifié (44\%) et en particulier secondaire et supérieur (27\% des personnes contre $15 \%$ ). Notons, de plus, que la majorité $(55 \%)$ touche des revenus, qu'ils soient sous forme de salaire ou d'autres ressources légales, une minorité $(7 \%)$ touchant des prestations sociales.

Ce sont, ensuite, les supports spécifiques de ces réseaux qui ressortent de ce corpus en termes de sexe et d'origine. Bien que la prédominance des hommes soit une constante, davantage de femmes sont impliquées dans les réseaux de trafic où elles jouent des rôles actifs. Elles ne sont pas seulement des intermédiaires passives (faire des commissions, passer des messages ou entreposer des sacs) ni réduites à des rôles périphériques (passer des messages ou des marchandises, blanchir l'argent, etc.). Elles interviennent aussi dans les différentes étapes du trafic où elles font valoir leur compétence (voyager à l'étranger, réceptionner les convoyeurs et les marchandises, négocier les transactions, etc.). La féminisation des réseaux s'appuyant sur ces compétences est un phénomène récurrent, qui se trouve confirmé sur d'autres terrains ${ }^{13}$. Bien que ces catégories soient insuffisantes pour cerner les dimensions ethniques des trafics, on constate, par ailleurs, une part importante d'étrangers (47\%), majoritairement d'Europe, puis des pays du Maghreb à égalité avec l'Amérique, qui contraste avec la faible part de personnes en situation irrégulière $\left(3 \%\right.$ contre $45 \%$ dans les affaires de trafic loca $\left.{ }^{14}\right)$. La nature de la drogue est bien évidemment discriminante, une plus forte proportion de personnes originaires du Maghreb étant impliquée dans les réseaux de cannabis, alors que les réseaux d'héroïne sont davantage mixtes sur le plan de l'origine culturelle.

Enfin, le statut pénal laisse apparaître une forte proportion de personnes ayant des antécédents (58\% en moyenne) et des casiers judiciaires chargés. Si $60 \%$ ont des antécédents en matière de stupéfiants (contre $69 \%$ pour le corpus d'affaires locales), on remarquera que $6 \%$ seulement sont déclarées usagères (contre $48 \%$ ). On compte aussi une proportion significative d'anciens braqueurs (16\% font état de vols à

13. Voir, par exemple, l'enquête de Fagan (1995) sur deux quartiers de Washington.

14. Il est vrai que la présence sur le territoire de la juridiction de l'aéroport RoissyCharles de Gaulle constitue un biais dans la mesure où y sont interpellés des «passeurs » d'origine étrangère, dont les policiers tirent peu de choses sur les structures dans lesquelles ils agissent. 
main armée). Parmi eux, on observe des variations significatives selon qu'ils sont impliqués dans les trafics d'héroïne (69\%), de cannabis (14\%) ou de cocaïne (6\%). Par contre, l'héroïne enregistre la plus forte proportion de personnes ayant des antécédents (69\%).

Il résulte de ces données des profils davantage intégrés socialement, des réseaux plus féminisés et cosmopolites, et des formes de délinquance différenciées, par comparaison aux trafics locaux étudiés. Ces constats conduisent à interroger, d'un point de vue plus qualitatif, les conditions sociales de l'engagement dans des réseaux de trafics d'envergure. Quelles sont les logiques dominantes sur lesquelles repose cet engagement? Tirer un profit financier semble être un motif décisif, et c'est sur lui que repose la qualification pénale. Reste à savoir dans quel objectif: pour faire vivre sa famille, sortir d'un endettement, acquérir des biens de consommation inaccessibles, s'enrichir?

Il faudrait consacrer un long développement aux trajectoires délinquantes et aux carrières dans ce type de trafic ${ }^{15}$. Pour aller à l'essentiel, disons que l'on trouve dans ces réseaux deux cohortes bien distinctes. D'un côté, celle des années 1960 : elle comprend davantage de personnes ayant une scolarité qui leur permet d'exercer une activité professionnelle. Les effets conjugués de la crise de l'emploi et du désintérêt à l'égard du métier expliquent que ces individus aient pu trouver dans des activités criminelles une alternative. Ainsi, la part de ceux qui présentent des antécédents lourds (crimes et délits aggravés) et fortement pénalisés l'emporte largement dans notre corpus sur le taux de récidive pour infraction à la législation sur les stupéfiants. D'un autre côté, il y a la cohorte des personnes nées dans les années 1970 dont la scolarité est chaotique et qui peinent à amorcer un cursus professionnel. Ces individus vont d'occupations précaires en combines dont l'enchaînement précipite leur carrière délinquante. Parmi ces activités, celles liées à la revente de stupéfiants occupent une place importante dans un contexte où l'explosion du business semble proportionnelle aux processus de désaffiliation à la fois sociale, scolaire, urbaine et institutionnelle (Kokoreff, 2000). Si les populations présentant des situations plus intégrées socialement interviennent davantage dans la «partie haute» des réseaux, c'est qu'elles sont à même de mobiliser des compétences particulières ou des activités professionnelles qui les protègent des processus de margina-

15. C'est l'objet de la troisième partie du rapport cité plus haut (Duprez et al., 2000 : 295-338). 
lisation sociale. On a donc affaire à des individus dont les trajectoires sociales ascendantes sont traversées par des logiques économiques (concurrence, hiérarchie, exploitation des plus faibles) et qui maintiennent un statut social par leur engagement dans ces milieux illicites.

\section{L'affaire P., prototype du réseau en bande organisée?}

Comment fonctionnent ces réseaux? Quel est leur degré d'organisation? Quelle place accorder aux dimensions proprement sociales? Nous nous appuierons sur une étude de cas pour suggérer quelques réponses. Il s'agit d'une importante association internationale de trafiquants dont 36 membres ont été interpellés et placés en mandat de dépôt. Du fournisseur installé sur la Costa del Sol au semi-grossiste parisien écoulant par dizaines de kilos, en passant par les livreurs dont certains faisaient trois rotations Fuengirola-Paris-Fuengirola en quinze jours, apportant $125 \mathrm{~kg}$ de cannabis pour repartir avec, dans les mêmes caches, des millions de francs en petites coupures; de l'homme de main chargé de la récupération de la marchandise arrivant du Maroc par 400 ou $500 \mathrm{~kg}$ au garagiste qui a aménagé les caches pour une demi-douzaine de véhicules, sans oublier les revendeurs chargés d'écouler - parfois sans succès - des quantités allant de quelques kilos à quelques dizaines de kilos de résine de cannabis, il s'agit d'une véritable filière d'approvisionnement à l'échelle du territoire national.

Le principal organisateur de ce réseau (on l'appellera «Paulo») fait partie des anciens d'un groupe (la «bande de la banlieue sud») qui a connu ses heures de gloire dans les années 1970 avec de multiples braquages. Son casier judiciaire mentionne entre 1972 et 1992 six condamnations autres que pour des infractions liées aux stupéfiants (homicide involontaire, vol, recel, falsification de chèques). Le réquisitoire définitif date du début des années 1990, le changement de cap de «Paulo» correspondrait à une modification de la législation sur les machines à sous, soumises à l'impôt. C'est en effet en 1992 qu'il est condamné par défaut à 12 ans de réclusion par la justice espagnole pour importation de stupéfiants dans le cadre d'une des premières affaires internationales de résine de cannabis. Sa fuite de l'Espagne lui permet d'échapper à cette peine. En vérité, sa reconversion dans une activité jugée plus lucrative date du début des années 1980. Il commence modestement, négocie en personne avec les producteurs dans les zones montagneuses du Maroc l'achat de cannabis qu'il importe par centaines 
de kilos. Connu dès cette période des services de police et de la justice, il bénéficie de plusieurs non-lieux. "Paulo» passe ensuite à une phase plus organisée. En attestent les livraisons reconnues par les personnes mises en examen. La distribution se situe à l'échelle nationale, dans la région parisienne, à Paris, Chartres, Bordeaux, Lyon et Toulouse. Quatre dépôts sont localisés, dont trois en Seine-Saint-Denis et un sur la côte landaise. Sans trop rentrer dans les détails, disons que ce sont entre 53 et 58 livraisons de plus de $100 \mathrm{~kg}$ de cannabis qui ont été reconnues en totalité par les 4 chauffeurs incriminés!

Tout porte donc à penser que ce trafic était particulièrement lucratif. Pourtant, on sait peu de choses à propos de la rubrique «prix, salaires et profits». Si on peut se faire une idée du chiffre d'affaires global, à partir des sommes récupérées quelques jours après la livraison par les passeurs, c'est dans des circonstances quelque peu surprenantes: deux chauffeurs se sont en effet fait voler dans leur véhicule l'un, 2,2 millions de francs, l'autre, près de 1 million. On sait que le troisième chauffeur prenait $1000 \mathrm{~F}$ de commission par kilo, ce qui pour des livraisons de $100 \mathrm{~kg}$ en moyenne lui rapportait $100000 \mathrm{~F}$ par passage, et il lui arrivait de faire trois passages par quinzaine. La perquisition effectuée en Espagne, au domicile de la mère du bras droit de «Paulo», permet de lui arracher des mains in extremis des documents manuscrits comptables. Ceux-ci portent sur des sommes détenues en livres sterling, francs suisses et français et en pesetas en compte courant, pièces d'or et billets d'un montant total de $6382000 \mathrm{~F}$.

Autre indice: alors que l'un des passeurs est incarcéré, «Paulo» demande à la compagne de celui-ci de récupérer l'argent des $125 \mathrm{~kg}$ qu'il a livrés. Avec l'aide de 3 personnes elles aussi impliquées dans le trafic, elle restitue $780000 \mathrm{~F}$. Mais faute de connaître avec précision le prix d'achat en Espagne de la marchandise et son prix de revente par les distributeurs, il est difficile de calculer les marges bénéficiaires des principaux acteurs du trafic. Selon les déclarations d'un revendeur, qui aurait écoulé $60 \mathrm{~kg}$ durant 6 mois, le kilo de résine de cannabis était acheté $11000 \mathrm{~F}$ - ce qui semble beaucoup - et revendu $12500 \mathrm{~F}$ - ce qui correspond davantage aux prix du marché ${ }^{16}$.

16. En 1996, les prix à la production étaient estimés par les policiers entre 2 et $3000 \mathrm{~F}$ le kilo, auxquels s'ajoutent l'acheminement vers les côtes marocaines (+ 1000 F) et l'exportation vers l'Espagne (+ $1000 \mathrm{~F})$, ce qui fait entre 4 et $5000 \mathrm{~F}$ le kilo. Dans les agglomérations, il sera revendu 9 à $13000 \mathrm{~F}$. Cela dit, on sait ce que ces projections ont d'aléatoire. Ainsi, tout dépend des qualités: la Haya (produit de qualité recherché) revenait à 6/7 $000 \mathrm{~F}$ en Espagne et pouvait être revendue entre 13 et $14000 \mathrm{~F}$. 
Des éléments plus probants concernent les opérations de blanchiment décrites assez précisément en ce qui concerne les trois personnes occupant un rôle stratégique dans le réseau du Maroc en Espagne, de la logistique vers la France et de la distribution dans la région parisienne. Ainsi, «Paulo» a ouvert plusieurs comptes bancaires au nom de sa fille, dans deux banques espagnoles. Il lui a offert un appartement et une somme d'un montant de $63040 \mathrm{~F}$. Il a participé à hauteur de $200000 \mathrm{~F}$ à l'achat d'une ferme par son fils. Au domicile de sa femme étaient saisis divers titres estimés à une valeur totale de $363610 \mathrm{~F}$. De son côté, «Momo», un de ses bras droits, a acquis des parts d'un hôtel au Maroc décrit comme "luxueux». Mais il semble que ces gains ont surtout été placés au nom de la femme de ce dernier. Pourtant, elle expliquera que son appartement de Courbevoie, son automobile, ainsi que des bijoux découverts dans son coffre estimés à $300000 \mathrm{~F}$, lui ont été offerts par son ancien amant, un milliardaire du Quatar. Aux dires des enquêteurs, elle ne pouvait pas non plus justifier l'origine des fonds nécessaires à l'acquisition d'un bar dont elle était gérante de droit, tombant ainsi sous l'inculpation de «proxénétisme de la drogue». Ces éléments illustrent la prééminence de l'assise familiale des trafics, assise qui offre bien des ressources en termes de socialisation des risques et de protection. Néanmoins, l'enrichissement constaté est loin de concerner l'ensemble des membres du réseau. Là comme ailleurs, les positions lucratives sont limitées; elles sont fonction des positions occupées dans le réseau et des rapports sociaux entretenus non seulement entre commanditaire, chauffeurs, distributeurs et revendeurs mais avec leurs proches, comme on va le voir.

\section{Les rapports sociaux de trafic}

Ce trafic s'appuie sur un réseau de relations anciennes. Le réquisitoire multiplie les mentions de liens durables s'articulant autour du principal organisateur du trafic. Si, du point de vue de l'accusation, ces relations peuvent confirmer l'existence d'une infraction en bande organisée et contredire a priori un système de défense des mis en examen montrant le caractère contingent de ces relations, d'un point de vue sociologique, elles attestent d'une "éthique des réseaux basée sur la confiance» (Tarrius, 1997: 109-112). Il y a là une dimension essentielle du fonctionnement des réseaux de l'économie informelle. D'ailleurs, à partir 
du moment où le travail d'enquête des services de police judiciaire commence à porter ses fruits, on constate une fragilisation du réseau, la méfiance succédant à la confiance.

C'est qu'il se passe de drôles de choses dans ce réseau: vols, impayés, violences et assassinat, mise en place de filières parallèles, etc. Les deux chauffeurs se font voler plus de trois millions de francs dans leur véhicule. «Paulo» soupçonne le premier d'en être l'auteur, mais ce dernier reprendra ses rotations. Soupçonné d'avoir collaboré avec les policiers, un «contrat» est lancé contre lui, auquel il échappera de justesse grâce à son interpellation par ceux-ci. «Paulo» ne confie plus de marchandises au second chauffeur mais le charge de récupérer l'argent, car il est réputé être mauvais payeur. Le grossiste marocain suspend toute relation avec un des distributeurs en région parisienne. En rupture de stock, celui-ci, basé en région parisienne, décide de monter une filière parallèle avec trois complices en important du cannabis de Belgique et de Hollande pour la France et l'Italie. Par ailleurs, certaines livraisons sont effectuées à l'insu du commanditaire. Deux autres personnages réalisent des livraisons en surcharge à Burgos, ainsi que des importations à destination de l'Angleterre. Le réquisitoire indique qu'une filière parallèle, à l'insu de son créateur affaibli, était en projet. Dans ce sens, dépitée par les infidélités conjugales de «Paulo», son épouse aurait aussi décidé d'utiliser pour son compte le réseau qu'il a mis en place. De fait, la dite filière continue de fonctionner après l'arrestation de son mari.

Et puis, il y a deux homicides. Le premier, en 1994, va bousculer le déroulement $\mathrm{du}$ dossier établi à partir d'une information d'un service de police à propos d'une transaction ayant conduit à l'interpellation d'un intermédiaire et d'une saisie de $86 \mathrm{~kg}$ de cannabis. Sur le corps de la victime, abattue froidement de quatre balles tirées à bout portant par un motocycliste casqué dans un restaurant, est retrouvée une coupure de presse relatant l'arrestation de cet intermédiaire. Simultanément, ce dernier demande à être entendu par le juge d'instruction. Il explique pour quelles raisons ce meurtre a eu lieu: la victime travaillait pour le même fournisseur et avait importé d'Espagne $100 \mathrm{~kg}$ sans passer par les intermédiaires habituels. N'ayant pu récupérer l'intégralité du prix de la marchandise livrée, il avait dû rendre des comptes, avant d'être abattu pour ne pas avoir payé le reliquat. Le second, survenu en 1995, est plus obscur : il s'agit d'un des principaux contacts de «Momo» tué par un jeune revendeur de 26 ans au moment des faits, sans doute aussi sur ordre. 
Ces différents éléments sur les pratiques suggèrent que l'on a, certes, affaire à un type d'organisation assez structuré dans sa logistique pour rendre possible des passages réguliers et approvisionner plusieurs métropoles françaises. Les caractéristiques des profils et trajectoires des différents acteurs de ce trafic, les compétences et savoir-faire qui sont les leurs, leurs antécédents judiciaires, peuvent se comprendre comme autant d'éléments explicatifs de ces formes professionnalisées. Cela étant, il fait aussi ressortir l'hétérogénéité d'un « réseau» qui réunit, en fait, divers niveaux ou strates, ce qui expliquerait, au moins en partie, les tensions, conflits et violences qui le caractérisent. C'est dans cette perspective qu'il nous faut maintenant aborder l'analyse des pratiques de trafic telles qu'elles sont appréhendées par les policiers et les magistrats et traduites dans le cadre de la procédure criminelle.

\section{La construction des pratiques par la machine pénale}

La procédure criminelle marque une évolution remarquable du traitement pénal du trafic de stupéfiants. Par ces dispositions, le législateur a prévu les peines les plus graves pour les trafics perpétrés dans la cadre de la criminalité organisée. Telle était la logique générale de la réécriture du code pénal dépassant le cas particulier des stupéfiants : clarifier les règles en matière d'échelle des peines dans le droit commun. La principale innovation de ce texte est l'indexation de l'échelle des peines en fonction non plus des risques que le trafic fait courir aux victimes potentielles, mais de l'importance et du degré d'organisation de cette activité illicite ${ }^{17}$. Dès lors, il ne suffit pas que soit établi un mouvement frontalier; encore faut-il que soit mise à jour la concertation de plusieurs personnes en vue d'un dessein criminel avéré. Un autre point important concerne l'instance chargée de l'audience: il s'agit d'une cour d'assises spéciale composée de sept magistrats spécialisés. Les cours d'assises spéciales ont été instaurées en France, en 1986, à la suite du procès des membres d'Action directe. C'est donc le terrorisme qui sert de modèle.

Si on considère la mise en œuvre dans l'espace local de cette procédure, l'enquête révèle l'bétérogénéité des pratiques selon les parquets ${ }^{18}$. Ainsi,

17. Voir sur ce point les commentaires éclairants d'Aubusson de Cavarlay (1999).

18. Pour une analyse des politiques pénales «locales», voir Kokoreff (2001). 
au tribunal de Bobigny, ce sont plus d'une dizaine d'affaires qui ont été ouvertes au criminel; mais plusieurs ont été requalifiées en délits correctionnels, et dans un cas, le tribunal a considéré son incompétence pour renvoyer l'affaire en cour d'assises spéciale. Au tribunal de Nanterre, trois affaires ont été d'entrée de jeu criminalisées, mais notons que deux autres affaires d'importation importantes sont restées correctionnalisées. Enfin, au tribunal de Lille, aucune affaire n'avait été, au moment de l'enquête, ouverte avec cette qualification, certaines affaires ayant été renvoyées à d'autres juridictions afin d'éviter l'engorgement, alors que d'autres, traitées par cette juridiction, auraient très bien pu être criminalisées.

Peu de juridictions ont mis en œuvre cette procédure. La raison en est simple: il s'agit d'affaires qui, de par leur nature, le nombre de personnes impliquées, la configuration des réseaux, renvoient à des procédures complexes (recompositions des qualifications, nombre élevé de renvois, disjonctions, nombre de personnes, etc.) et lourdes (commissions rogatoires innombrables, y compris sur le plan international, ouverture d'informations complémentaires, demandes d'extradition, etc.). Les délais se trouvent amplifiés par des coopérations internationales toujours incertaines, pour des raisons liées aux différences de procédures (c'est le cas de l'Espagne) mais aussi politiques (c'est le cas du Maroc ${ }^{19}$ ). Il en résulte des dossiers volumineux (certains comprennent plusieurs dizaines de tomes). Ainsi, par exemple, un magistrat nous expliquera à propos d'une autre affaire d'importation de cannabis: "C'est un dossier assez volumineux, avec pas mal de personnes qui apparaissent. C'est un dossier qui n'est pas très complexe mais où il $\mathrm{y}$ a beaucoup de personnes et où il fallait se repérer: c'est le problème de ce type de dossiers». D'où la réticence de certains parquets généraux, craignant la lourdeur de cette procédure et l'effet d'engorgement du tribunal occasionné par la mobilisation de sept magistrats du parquet pendant un procès qui peut durer plusieurs semaines.

Mais ce sont aussi des logiques policières qu'il convient de prendre en compte. À l'origine de l'affaire P., on l'a dit, il y a une importante saisie de cannabis. Le parquet est saisi et ouvre une instruction. Le juge demande de poursuivre ses investigations à la brigade qui a réalisé

19. Tout se passe comme si l'État français et la communauté européenne avaient passé avec l'État marocain un accord faisant de la lutte contre le terrorisme islamiste une priorité par rapport à la production de cannabis et à ce qu'elle rapporte. 
l'interpellation. Mais ce service se montre peu enthousiaste. C'est la logique quantitative qui prime ("plus on saisit de kilos, mieux on travaille»), et dans ce cas, le résultat (près de $100 \mathrm{~kg}$ de résine de cannabis) est probant. Un service départemental de police judiciaire est alors contacté; comme l'affaire ne leur a pas été confiée au départ, ses membres sont peu motivés mais obtempèrent. L'affaire s'enlise. C'est la fusillade survenue dans un restaurant qui conduit le juge à dessaisir ce service du dossier et à en saisir la brigade des stupéfiants de la DRPJ (Direction régionale de police judiciaire) qui enquête sur ce meurtre. Plus généralement, ces affaires sont ouvertes au criminel non pas seulement du fait des circonstances aggravantes de l'infraction mais eu égard à son caractère organisé, avec ce que cela suppose comme lien avec le grand banditisme, le proxénétisme ou les systèmes mafieux. Ce qui explique que ces affaires soient traitées par des services spécialisés qui disposent de moyens (financiers, humains, technologiques) et de compétences correspondant à leur mission: la répression du trafic de stupéfiants et de la criminalité organisée à l'échelle internationale. Dans le cas de l'affaire P., l'essentiel du travail policier va reposer sur l'exploitation des écoutes et des interceptions téléphoniques, ce qui le différencie des enquêtes portant sur d'autres formes de trafics où, bien souvent, l'essentiel de l'activité policière consiste à mettre en place des dispositifs de surveillance, à accumuler les dépositions des usagers interpellés, quand elle ne se limite pas à faire du «saute-dessus» pour reprendre l'expression consacrée des policiers en matière de flagrant délit.

\section{Le modèle hiérarchique et ses limites}

La logique qui sous-tend la construction pénale des affaires implique un certain nombre de principes qui sont autant de «biais» dans l'analyse qu'on peut en faire. Ainsi, au sein des dossiers judiciaires, le réquisitoire définitif est une pièce essentielle : en effet, ce document donne forme à l'ensemble de l'affaire, de sorte qu'elle est en état d'être jugée. Dans l'affaire P., les filières seront décomposées en cinq niveaux : 1) la «création de la bande organisée»; 2) les «clients-grossistes en France»; 3) les «chauffeurs-livreurs»; 4) les «dépositaires de drogues»; 5) les «revendeurs».

Poser les relations en termes hiérarchiques peut se comprendre comme un élément parmi d'autres d'une routine consistant à resituer les protagonistes d'une affaire les uns par rapport aux autres. Mais cela s'inscrit 
aussi dans une logique de la poursuite qui est celle du pénal, la présentation de la filière selon un modèle hiérarchique facilitant les qualifications imputables à chacun des individus mis en examen. Or, fonder une logique de la peine ne va pas de soi et ne produit pas toujours les résultats attendus. On en veut pour preuve que, lors du jugement en septembre 2003, le commanditaire du trafic («Paulo») a été condamné à 10 ans de prison, le principal distributeur dans la région parisienne à 8 ans, alors que les «hommes de main», "passeurs» et autres ont été condamnés à des peines inférieures ou égales à 5 ans. Si les peines rendues n'ont pas été à la hauteur des réquisitions du parquet, selon l'explication donnée par l'avocat général, c'est au regard de la nature du produit («ce n'est que du cannabis» répéteront les avocats de la défense) présentant une dangerosité moindre que d'autres drogues!

Qui dit trafic en bande organisée suppose de mettre en relief une organisation structurée se traduisant dans les représentations par un modèle pyramidal. Or, on l'a vu, les relations ne sont pas seulement hiérarchiques. Elles mettent en présence de «vieux amis», sans parler de relations de détention qui impliquent un type de lien spécifique. Par ailleurs, il y a dans cette organisation des courts-circuits, des filières parallèles qui émergent, de la violence, des adultères et des vengeances. Enfin, les positions sont mobiles, surtout dès lors que l'action policière commence à faire sentir ses effets : tel homme de main se retrouve livreur, les clients-grossistes sont, à l'occasion, des chauffeurs. On a davantage affaire à des grappes ou des cliques qu'à une structure hiérarchique.

Dans une autre affaire présentant des caractéristiques similaires et datant de la même période, celui qui apparaît comme la «tête de réseau» côté français est aussi un ancien de la «bande de la banlieue sud» qui a commis nombre de braquages dans les années 1970. Partie d'un coup de téléphone anonyme signalant aux policiers l'existence d'un réseau de revente de cannabis depuis un bar, cette affaire va conduire à la révélation d'un réseau, avec ses ramifications étrangères, et à la mise en cause de près de cinquante personnes. Les flux de marchandises sont, ici encore, considérables, allant de $400 \mathrm{~kg}$ à 6 tonnes perdues lors d'un transport en mer. Dans cette affaire, pour reprendre la formule du magistrat instructeur, "l'organisation était en amont», de la tête de réseau à l'intermédiaire marocain en Europe. «Le reste était organisé de façon artisanale, à la va-comme-je-te-pousse».

Les professionnels se situent plutôt du côté des commanditaires. C’est le cas de celui prénommé «Nono la Puce», commanditaire pré- 
sumé du premier bateau dont la cargaison de trois tonnes échouera dans l'océan, à la suite de l'intervention de la garde civile espagnole. Considéré par le magistrat instructeur comme étant peut-être l'un des plus grands truands en France, il a été arrêté en Espagne pour une affaire d'importation de 13 tonnes de cannabis. Mais il a également été poursuivi à l'époque pour une affaire de triple assassinat au fusil mitrailleur à Marbella, sans parler du triple mandat d'arrêt dont il a été l'objet en France pour attaque de fourgons blindés, meurtre et trafic en bande organisée. Cette figure du grand banditisme témoigne du niveau de violence dans ce type d'organisation. D'ailleurs, le magistrat nous expliqua que, bien qu'il n'y ait pas eu de préjudice puisqu'il n'y a pas eu d'avance, les membres du clan organisateur du trafic ont pris peur; ils ont été obligés de déménager, "obligés de faire venir des outils, c'est-à-dire des armes, de France, parce qu'ils [auraient] peur d'un règlement de compte à grande échelle ».

Si tous les membres du réseau ne disposent pas de compétences égales, à l'inverse, on trouve dans les trafics locaux certaines caractéristiques de ces trafics en réseau: division du travail accrue, professionnalisme des «grosses têtes» - (pour reprendre cette catégorie indigène) se traduisant dans la distribution, l'embauche et la revente - opérations de blanchiment bénéficiant des ressources des réseaux familiaux et d'interconnaissances, climat de peur et de violence entourant les lieux de revente et les «petites mains». Ainsi, par exemple, certaines affaires révèlent que le trafic fonctionne parfois comme une concession. Loin d'entrer dans des rapports de concurrence desservant les intérêts de tous, plusieurs équipes se partagent un territoire. Trois équipes comprenant chacune trois ou quatre personnes travaillent à tour de rôle, bénéficiant d'un accord tacite entre elles, à raison d'une rotation tous les mois selon les uns, toutes les semaines selon d'autres. L'adoption des techniques classiques de la clandestinité qui consistent à utiliser pour certaines opérations, à leur insu, des individus au-dessus de tout soupçon, peut aussi être un indicateur de professionnalisation des trafics dans les cités et quartiers pauvres. C'est aussi à cet égard que la distinction entre trafic international et trafic local est «flottante».

\section{La coalition des «milieux»}

La construction de ces affaires autour de la notion de bande organisée ne doit pas dissimuler, nous l'avons souligné, l'appartenance de ses 
différents protagonistes à des milieux sociaux hétérogènes. Dans un premier temps, elle révèle l'inscription du trafic dans les milieux « traditionnels» de la délinquance: d'une part, ceux du banditisme et du proxénétisme, d'autre part, ceux qui se déploient à partir des casses d'automobiles et des ferrailleurs. Mais il apparaît aussi qu'un certain nombre d'individus, dont le rôle est certes subalterne dans le trafic, ne peuvent être assimilés à ces milieux. L'audience correctionnelle qui a eu lieu en mai 2000 est intéressante à cet égard. De cette femme de cinquante ans, intérimaire, qui aurait écoulé près de $25 \mathrm{~kg}$ de cannabis pour faire face à ses difficultés financières, à ce gérant d'un hôtel restaurant poursuivi pour avoir procédé à plusieurs livraisons en acquittement d'une dette, en passant par ce jeune magasinier contraint par son frère en situation illégale sur le territoire français et par ce patron de pizzerias dans les Landes qui accompagne le troisième chauffeur dont il se sent l'obligé dans ses livraisons vers Paris, on a affaire à des «seconds rôles» dont on peut penser qu'ils ne savaient pas dans quel type de trafic ils étaient impliqués. C'est le travail d'investigation de la police qui donne sa consistance au réseau. D'ailleurs, fait significatif: un seul homme comparaissait dans le box des détenus, après avoir été condamné à 20 ans de réclusion pour homicide. Comme le dira le commandant de la brigade des stupéfiants de la DRPJ chargé de l'enquête à l'audience: il s'agit d' «un voyou qui gère sérieusement ses affaires; les autres ne jouent pas dans la même catégorie». L'audience confirmera ce constat, y compris dans le délibéré, avec des condamnations allant de 6 mois à 2 ans fermes pour les 11 prévenus.

Tout le travail de construction par la machine judiciaire consiste donc, là comme ailleurs, à donner une cohérence à un ensemble de faits retranscrits dans la logique du système pénal, quitte à «saucissonner » le dossier - avec néanmoins cette spécificité qui tient au nombre de personnes mises en examen et à leur mode de relation qui s'appuie moins sur des supports territoriaux que sur des réseaux d'interconnaissances délocalisés. On comprend mieux, dans ce sens, le rôle central de la prison. Les documents étudiés ne manquent jamais de rappeler que tel et tel prévenus se sont rencontrés en détention. Tout se passe comme si ce fait confirmait les activités délictueuses des protagonistes, illustrant le constat banal selon lequel la prison est l'école du crime. Un autre phénomène réside dans la référence à un ethos commercial. Entre ces individus, il n'est question que de business, d'argent, d'investissement. Ce 
sont des commerçants, pas des consommateurs, à l'exception de deux ou trois personnes.

\section{Conclusion}

L'intérêt de prendre les formes les plus professionnalisées de trafics comme objet sociologique est de faire apparaître un double mouvement. D'un côté, le fait majeur de notre enquête sur les juridictions de Bobigny et de Nanterre est la reconversion du grand banditisme dans le trafic de drogues, d'abord de cannabis dans les années 1980, puis de cocaïne au cours de la décennie suivante. Cette reconversion a-t-elle été rendue possible par l'affaiblissement des cartels colombiens et des mafias italiennes? S'inscrit-elle dans l'émergence d'une nouvelle économie criminelle mondialisée? Toujours est-il que les trajectoires sociales des braqueurs, proxénètes, truands, la violence professionnelle qui apparaît dans les dossiers, les connexions élargies à d'autres marchés et secteurs de la criminalité organisée, constituent des indicateurs de ce processus. En conséquence, un tel processus invite à renouveler les catégories d'analyse. Les affaires étudiées incitent à nuancer la conception pyramidale et centralisée des organisations criminelles qui prévaut encore largement aujourd'hui. Si l'exemple des réseaux d'importation met en relief une forte division verticale du travail et des rôles à une large échelle, il ne s'agit pas d'en surestimer l'importance. On a vu que les hiérarchies étaient dans certaines circonstances flottantes et que les acteurs dans ce trafic ne constituaient pas un «milieu» homogène mais appartenaient à des mondes hétérogènes. Cette «hétérogénéité désorganisante » peut constituer une source supplémentaire de tensions liées à toute une série d'irrégularités. Certes, on pourra toujours expliquer par là le repérage et le démantèlement de ces réseaux. Mais le fait est que ces derniers s'inscrivent aussi dans une économie de l'incertitude dont il importe de saisir la dynamique. On en veut pour preuve le fait que les marchés des grossistes, du côté du sud de l'Espagne, et ses distributeurs, notamment en région parisienne, auraient changé de main depuis la fin des années 1990, à la suite d'une série de règlements de compte. Une autre tendance manifeste concerne la multiplication des passages de frontière à partir des points de vente locale. Ainsi, dans le département où nous avons travaillé, les policiers estimaient entre 500 et $800 \mathrm{~kg}$ les quantités de cannabis passées chaque semaine par de 
nombreuses voitures venant approvisionner les marchés locaux. Là où règne une vision du commerce local dominée par la désorganisation, la dispersion, la précarité, on est amené à s'interroger sur de telles représentations. Si elles ne sont pas sans fondement en ce qui concerne certains secteurs, elles ne doivent pas masquer des formes de trafics beaucoup plus organisées. Ignorer ces dynamiques en continuant d'utiliser des catégories statiques, c'est occulter ces emboîtements entre l'international et le local, et au sein du local à divers échelons, et par là, renforcer l'injustice de la stigmatisation pesant sur les quartiers dits «sensibles».

D'un autre côté, ces formes nous paraissent un bon révélateur de l'évolution des politiques pénales et de leurs contradictions. On l'a vu, les affaires étudiées sont d'abord une construction pénale, structurées qu'elles sont à partir d'une logique de la poursuite qui souligne leurs limites. Mais elles sont aussi le produit d'une politique publique. Les nouvelles qualifications pénales visent à sanctionner les infractions les plus graves. Au regard des réformes les plus récentes de la procédure pénale, on constate que la répression des trafics de stupéfiants fait office de modèle pour lutter contre tout un ensemble de délits et de crimes en «bande organisée $e^{20}$ ». Or, le paradoxe est pourtant là : le but était de réprimer mieux, le résultat est de peu réprimer à ce niveau d'infraction. On en veut pour preuve les fréquentes requalifications en cours d'instruction; certaines affaires peuvent être «recorrectionnalisées» par exemple. En outre, les condamnations ne sont pas à la hauteur des réquisitions. Est-ce faute d'avoir défini une politique globale pour ce type de répression? Fallait-il spécialiser la procédure d'enquête et d'instruction, créer une cour d'assises spéciale pour cour d'appel, comme l'ont demandé certains syndicats en France? Sans doute. Mais il faudrait ajouter les effets de logiques et de cultures professionnelles bien établies: l'éparpillement des enquêtes sur de nombreux ressorts, le manque de coordination opérationnelle entre les services, une logique inflationniste qui se soucie plus d'augmenter les saisies que de s'attaquer à de vastes réseaux. Du coup, on peut s'interroger sur l'efficacité des politiques sécuritaires mises en œuvre depuis plus d'une dizaine d'années et leurs présupposés idéologiques.

20. La loi Perben sur la «criminalité organisée» en est la traduction la plus récente en France. Votée en 2003 après bien des débats et des contestations des professionnels du monde judiciaire, elle contribue à étendre les dispositions d'exception du trafic de stupéfiants contenues dans le nouveau code pénal à toute une série de crimes et délits. 
La lutte contre le trafic de stupéfiants, que ce soit sur le plan international, national ou aux divers échelons du local, est considérée par tous comme une priorité. La politique menée par les États-Unis est emblématique à cet égard, tant la «guerre à la drogue» a connu un regain d'activité dans les années 1980 pour lutter contre le commerce de crack et de cocaïne, ainsi que contre la criminalité qui y est associée. Tel a été le modèle à partir duquel les conventions internationales ont été élaborées et ratifiées par la plupart des pays occidentaux pour faire face aux narcotrafiquants. Or, force est de constater que cette politique n'a pas eu les résultats escomptés. Depuis le milieu des années 1990, tous les rapports des instances internationales et des experts attestent que les productions sont en hausse sur le plan mondial dans le cadre d'une économie de la drogue largement recomposée au sein d'organisations de plus en plus décentralisées ${ }^{21}$. Par ailleurs, la banalisation des consommations, en particulier de cannabis et de drogues de synthèse, est un fait bien établi par les enquêtes en population générale. Faut-il en conclure que l'objectif annoncé de l'action publique («démanteler les filières», lutter contre le «crime organisé») relève davantage d'un registre symbolique que d'une politique définie par des priorités et des choix? Ou bien les politiques publiques pêchent-elles par leur incohérence en même temps qu'elles achoppent sur une professionnalisation accrue des économies criminelles? C'est un débat qu'il faudrait réouvrir en s'appuyant sur des résultats de recherche plutôt que sur des postures idéologiques.

21. Ainsi, Alain Labrousse (2004) rapporte qu'en Colombie, aux trois ou quatre grands «cartels» se sont ajoutés entre 50 et 100 organisations moyennes et près de 3000 «petits entrepreneurs». De même, on comptait une douzaine de groupes de la Cammora à Naples; ils seraient aujourd'hui près d'une centaine. 


\section{Note méthodologique: le temps pénal et le temps de la recherche}

Il ne nous a pas été possible d'accéder au dossier de l'affaire P., pour des raisons de procédure qu'il n'est pas sans intérêt de relater. Au moment où nous avons commencé notre travail d'enquête, en 1998, le juge d'instruction estimait avoir quasiment terminé cette affaire. Il a transmis l'ensemble du dossier au parquet afin qu'il prenne des réquisitions en vue de la rédaction du réquisitoire définitif, ce qu'il a fait, estimant à son tour que le dossier était terminé. Ensuite, le dossier allait à la chambre d'accusation qui se devait d'apprécier, comme dans tout dossier criminel, si elle décidait un renvoi devant la cour d'assises, si elle prononçait un non-lieu, ou bien si elle ordonnait un supplément d'information, estimant que l'affaire n'était pas en état d'être jugée par la cour d'assises spéciale. Autre possibilité, elle pouvait considérer que les circonstances aggravantes n'étaient pas constituées et renvoyer ce dossier devant le tribunal correctionnel. En l'espèce, elle ordonna un supplément d'information, notamment pour réentendre le principal prévenu de façon plus précise sur les faits et éventuellement le confronter aux autres protagonistes de l'affaire. Étant détenu en Espagne où il purgeait une peine de six ans pour trafic de stupéfiants, la demande fut faite à la justice espagnole afin d'obtenir un prêt de détenu. Cette procédure prend du temps, et ce n'est qu'au début de l'année en cours que finalement celle-ci refusa cette demande. Il s'est donc agi pour le juge d'instruction de mettre en place une commission rogatoire internationale afin d'entendre le prévenu en Espagne et de répondre à la demande de la chambre d'accusation.

C'est pourquoi l'affaire a mis plus de temps que prévu à venir en audience. C'est aussi ce qui explique l'échéancier judiciaire qui a conduit à «saucissonner » le dossier, selon l'expression d'un avocat. En effet, un des prévenus a été jugé en cours d'assises pour homicide volontaire lié au trafic de stupéfiants et condamné à 20 ans de réclusion; sur les 37 personnes ( 29 hommes et 8 femmes) mises en examen pour l'affaire qui nous intéresse, la moitié a été placée en mandat de dépôt correctionnel pour être jugée par le tribunal correctionnel 11 mois après, alors que l'autre moitié a été placée en mandat de dépôt criminel.

Déjà ces éléments renseignent sur la complexité du dossier. Sur le plan méthodologique, l'analyse de ce dossier comprenant 23 tomes d'actes divers et de procédures aurait été sans doute extrêmement difficile à réaliser. Par contre, nous avons eu la possibilité d'étudier le réquisitoire définitif du parquet. Son volume (plus de 120 pages) autorise une première analyse de cette affaire. Par ailleurs, nous avons rencontré à plusieurs reprises le magistrat qui a instruit ce dossier pendant trois ans et le substitut qui a rédigé le réquisitoire définitif. De plus, nous avons assisté à l'audience de la $12^{\mathrm{e}}$ chambre du TGI de Nanterre qui traitait de la partie «correctionnalisée» du dossier. Contact a été pris avec deux avocats à l'issue de celle-ci. Enfin, nous avons pu recontrer le responsable de l'enquête policière et son adjointe à la brigade des stupéfiants de la Direction régionale de la police judiciaire de Versailles. C'est le croisement de ces différentes sources qui autorise une analyse tant des trafics que de leur traitement pénal. 


\section{Références}

Adler, P. (1985). Wheeling and dealing : an ethnograpby of an upper-level drug dealing and smuggling community. New York: Columbia University Press.

Aubusson de Cavarlay, B. (1999). Du dossier de procédure aux filières pénales, l'effet entonnoir et le syndrôme du réverbère. In C. Faugeron (ed.), Les drogues en France (151-159). Genève: Georg.

Bachmann, C., \& Coppel, A. (1989). Le dragon domestique. Paris: Albin Michel.

Boltanski, L., \& Chiappello, E. (1999). Le nouvel esprit du capitalisme. Paris: Gallimard.

Bouhnik, P., \& Joubert, M. (1992). Économie des pratiques toxicomaniaques et lien social. Dépendances, 4 (3), 25-36.

Bourgois, P. (1992). Une nuit dans une shooting gallery. Enquête sur le commerce de la drogue à East Harlem. Actes de la recherche en sciences sociales, 94, 59-78.

Bourgois, P. (2001). En quête de respect. $2^{\mathrm{e}}$ ed. Paris: Le Seuil.

Castells, M. (1999). Fin de millénaire. Paris: Fayard.

Colombie, T., Lalam, N., \& Schiray, M. (2000). Drogue et techno. Paris : Stock.

Duprez, D., \& Kokoreff, M. (2000). Les mondes de la drogue. Paris: Odile Jacob.

Duprez, D., Kokoreff, M., \& Weinberger, M. (2001). Carrières, territoires et filières pénales. Paris: OFDT.

Fagan, J. (1995). Women's careers in drug use and drug selling. Current Perspectives on Aging and the Life Cycle, 4, 155-190.

Faugeron, C., \& Kokoreff, M. (1999). Les pratiques sociales des drogues. Éléments pour une mise en perspective des recherches en France. Sociétés contemporaines, 36, 5-18.

Fernandez, L. (2002). Acteurs et territoires «psychotropiques»: ethnographie des drogues dans une périphérie urbaine. Déviance et société, 26 (4), 427-442.

Jamoulle, P. (2002). La débrouille des familles. Bruxelles: De Boeck.

Kokoreff, M. (2000). Faire du business dans les quartiers. Déviance et société, 24 (4), 403-424.

Kokoreff, M. (2001). L'incertitude des politiques pénales. L'exemple du contentieux de stupéfiants. Cahiers lillois d'économie et de sociologie, 35-36, 185-202.

Kokoreff, M. (2003). La force des quartiers. Paris : Payot.

Labrousse, A. (2004). De la production à la distribution des drogues. In M. Jauffret-Roustide (ed.), Les drogues. Approches sociologique, économique et politique (83-111). Paris: La Documentation Française.

Lacoste, J., \& Tremblay, P. (1999). De l'insertion sociale des marchés urbains de drogues prohibées: deux cas de figure nord-américains. Déviance et société, 23 (1), 41-58.

Letkermann, P. (1973). Crime as work. Englewood Cliffs, NJ : Prentice Hall.

Marks, D. (2000). Mr Nice, une autobiographie. Paris: Ed Mama.

Morselli, C. (2001). Structuring Mr. Nice: entrepreneurial opportunities and brokerage positionning in the cannabis trade. Crime, Law and Social Change, 35, 203-244. 
Observatoire géopolitique des drogues (1995). Géopolitique des drogues. Paris: La Découverte.

Peraldi, M. (2002). Cabas et containers. Activités marchandes informelles et réseaux migrants transfrontaliers. Marseille: Maisonneuve et Larose.

Preble, E., \& Casey, J. (1969). Taking care of business: the heroin life users in the street. International Journal of Addictions, 4 (1), 1-24.

Reuter, P., Mac Coun, R., \& Murphy, P. (1991). Money from crime. A study of the economics of drug dealing in Washington DC. Santa Monica: Rand Corporation.

Robert, P. (2002). L'insécurité en France. Paris: La Découverte.

Ruggiero, V., \& South, N. (1996). La ville de la fin de l'ère moderne en tant que bazar: marchés de stupéfiants, entreprises illégales et les «barricades». Déviance et société, 20 (4), 317-335.

Ruggiero, V. (1998). Trafics postfordistes et trafiquants «juste à temps». Les Annales de la recherche urbaine, 78 (mai), 89-94.

Tarrius, A. (1997). Fin de siècle incertaine. Perpignan: Ed Trabucaire.

Williams, T. (1990). Cocaine kids. Paris : Gallimard. 